Methods: New onset JDM children were randomised to receive either prednisone (PDN) alone or in combination with MTX or CSA. All children were given initially intravenous methylprednisolone, and then PDN starting with $2 \mathrm{mg} / \mathrm{kg} /$ day. Gradual tapering according to a specific protocol could lead to the safe dose of $0.2 \mathrm{mg} /$ $\mathrm{kg} /$ day by month 6 , then discontinued at month 24 . Major therapeutic changes (MTC) were defined as the addition or major increase in the dose of MTX/CSA/ other drugs or any other reasons for which patients were dropped from the trial. Patients were divided according to clinical remission (CR) $(C M A S=52$ and MD global=0 for 6 continuous months) into two major groups. Group 1 included those on CR, who could discontinue PDN, with no MTC (reference group). Group 1 was compared with those who did not achieve CR, without or with MTC (group 2 and 3, respectively). JDM core set measures (CSM) were compared within the 3 groups. We also calculated the gold standard group 1 median change in the CSM in the first 6 and over 24 months and applied a logistic regression model to identify predictors of $\mathrm{CR}$ with PDN discontinuation.

Results: 139 children were enrolled in the trial: 47 on PDN, 46 on PDN +CSA and 46 on PDN +MTX. We identified 30 (21.6\%) patients for group 1, $43(30.9 \%)$ for group 2 and $66(47.5 \%)$ for group 3 . At baseline all 3 groups had no differences in the CSM. Already in the first 2 months a clear differential trend in disease activity measures, according to clinical remission status and PDN discontinuation, could be identified. From the observation of the median change in the CSM of group 1 in the first 6 months, the following recommendations could be extrapolated: decrease corticosteroids from 2 to $1 \mathrm{mg} / \mathrm{kg} /$ day in 2 months if the MD-global, parent-global, CHAQ, DAS, CMAS, MMT or Phs measures have changed of at least $50 \%$; from 1 to $0.5 \mathrm{mg} / \mathrm{kg} /$ day in the following 2 months if the MD-global, CHAQ, DAS, CMAS show a change of at least $20 \%$; in the following 2 months (month 4-6) corticosteroids can be tapered up to the safe dose of $0.2 \mathrm{mg} /$ $\mathrm{kg} /$ day, if the disease activity measures remain at low/normal values. We finally ran a logistic regression model that showed that the achievement of PRINTO criteria 50-70-90 at 2 months from disease onset, an age at onset $>9$ years and the combination therapy PDN +MTX, increase the probability of clinical remission from 4 to 7 times (table 1 ).

Abstract OP0340 - Table 1 Logistic regression model for the outcome: achievement of remission (n/tot: 28/130; 21.5\%)

$\mathrm{OR}(95 \% \mathrm{CI}) \quad \mathrm{P}$

Responder at 2 months:

0.0076

Printo-50 ( $v$ s. not responder/Printo-20)

$5.41(1.37-21.32)$

Printo-70 (vs. not responder/Printo-20)

$6.90(1.91-24.99)$

Printo-90 (vs. not responder/Printo-20)

$4.46(1.08-18.38)$

Age at onset $>8.53$ years $(\leq 8.53$ years)

$4.64(1.69-12.71)$

0.0017

Treatment group: $\mathrm{PDN}+\mathrm{MTX}$ (vs $\mathrm{PDN} / \mathrm{PDN}+\mathrm{CSA})$

$3.63(1.30-10.09)$

0.0116

AUC of the model: 0.80

OR: Odds Ratio; 95\% CI: 95\% Confidence Interval; P\#. Likelihood Ratio test.

Conclusions: We propose evidence based specific cut-offs for corticosteroid tapering/discontinuation based on the change in JDM CSM of disease activity, and to identify the best predictors for clinical remission and corticosteroid discontinuation.

Disclosure of Interest: None declared

DOI: 10.1136/annrheumdis-2018-eular.3750

FRIDAY, 15 JUNE 2018

Triple T: T cells, technologies and therapies

\section{OP0341 INCREASED FREQUENCY OF CIRCULATING CD4 +CXCR5-PD1HI PERIPHERAL HELPER T (CTPH) CELLS IN PATIENTS WITH SEROPOSITIVE EARLY RHEUMATOID ARTHRITIS (RA)}

P. Fortea-Gordo ${ }^{1}$, L. Nuño ${ }^{1}$, A. Villalba ${ }^{1}$, D. Peiteado ${ }^{1}$, I. Monjo ${ }^{1}$, P. SanchezMateos $^{2}$, A. Puig-Kröger ${ }^{2}$, E. de Miguel ${ }^{1}$, A. Balsa ${ }^{1}$, M.E. Miranda Carus ${ }^{1}$. ${ }^{1}$ Rheumatology, Hospital La Paz - IdiPAZ; ${ }^{2}$ Immuno-oncology, Hospital Gregorio Marañon, Madrid, Spain

Background: A novel population of CD4 + T cells with B cell helping capacity has been described in the synovial tissues and peripheral blood of seropositive RA patients with an established disease, and termed 'peripheral helper' (Tph) cells. (Rao DA et al, Nature 2017) Tph cells are characterised by the lack of CXCR5 together with a bright expression of PD-1 (CD4 +CXCR5-PD-1 ${ }^{\text {hi }} \mathrm{T}$ cells). As opposed to CD4 +CXCR5+PD-1 ${ }^{\text {hi }}$ follicular helper T cells (Tfh), Tph cells are not located in lymphoid organs but accumulate in inflamed tissues. Tph cell numbers have not been previously examined in early RA (eRA).

Objectives: To study the frequency of circulating CD3 +CD4+CXCR5-PD- $1^{\text {hi }}$ Tph cells (cTph), in patients with eRA.

Methods: Peripheral blood was drawn from DMARD-naïve early RA patients (eRA) (2010 ACR criteria) with a disease duration $<24$ weeks $(n=42)$, and healthy controls $(\mathrm{HC})$ matched for age and gender $(n=42)$. For comparison, blood was also drawn from 66 patients with established RA (disease duration >2 years), 45 patients with Spondyloarthritis ( $\mathrm{SpA}$ ), and their age and gender-matched $\mathrm{HC}$ (one $\mathrm{HC}$ per patient). In addition, synovial fluid from 7 patients with established RA and 3 patients with SpA was examined. Established RA patients were receiving lowdose oral methotrexate and were naïve for biological agents. SpA patients were receiving NSAIDs, low-dose oral methotrexate and/or sulphasalazine and were naïve for biologicals. After isolation by Ficoll-Hypaque gradient, PBMCs were stained with antibodies to CD3, CD4, CXCR5, ICOS and PD-1, and examined by flow cytometry.

Results: The frequency of circulating CXCR5- cells gated for CD4 +T cells was not different among the studied groups. In contrast, eRA patients demonstrated an increased frequency of circulating CD4 +CXCR5-PD-1 ${ }^{\text {hi }}$ Tph and CD4 +CXCR5-PD-1 ${ }^{\text {hi } I C O S+T}$ cells. When examining seropositive (RF +and/or $A C P A+, n=25$ ) and seronegative eRA patients (RF- and ACPA-, $n=17$ ) separately, it was evident that the above described alterations were only apparent in seropositive eRA. Likewise, increased cTph numbers were observed in seropositive $(n=47)$ but not seronegative $(n=19)$ established RA, and not in SpA patients ( $n=45)$, which is consistent with data reported by Rao et al. Interestingly, this increased cTph cell frequency was observed only in seropositive RA patients with an active disease (DAS28 $>2.6, n=24$ ), whereas the numbers of cTph cells in established RA patients who had achieved remission (DAS28 <2.6, $n=23$ ) were not different from HC. Furthermore, Tph cells were present in the synovial fluid of seropositive RA $(n=4)$ but not of seronegative RA $(n=3)$ or $\operatorname{SpA}(n=3)$.

Conclusions: Tph cells may play an important role in the pathogenesis of seropositive but not seronegative RA. An increased cTph cell frequency is a marker of active, seropositive RA

REFERENCE:

[1] Rao DA, et al. Nature 2017;542(7639):110-114.

Disclosure of Interest: None declared

DOI: 10.1136/annrheumdis-2018-eular.3855

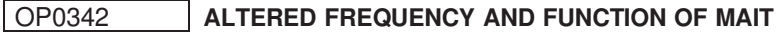 CELLS IN SYSTEMIC SCLEROSIS REVEALED BY HIGH DIMENSIONAL MASS CYTOMETRY AND TRANSCRIPTOME ANALYSIS}

B. Paleja ${ }^{1}$, A. Low ${ }^{2}$, P. Kumar ${ }^{1}$, S. Saidin ${ }^{1}$, A. Lajam ${ }^{1}$, L. D/O Thanna Bathi ${ }^{1}$, L. Lai ${ }^{1}$, C. Chua ${ }^{1}$, S. Albani ${ }^{1} .{ }^{1}$ Translational Immunology Institute; ${ }^{2}$ Department of Rheumatology and Immunology, Singapore General Hospital, Singapore, Singapore

Background: Systemic sclerosis (SSc) is an autoimmune disease characterised by excessive fibrosis of skin and internal organs, and vascular dysfunction ${ }^{1}$. Association of $\mathrm{T}$ and $\mathrm{B}$ cell subsets have been reported in SSc, however there is lack of systematic studies of functional relations between immune cell subsets in this disease $^{2,3,4}$. This lack of mechanistic knowledge hampers targeted intervention.

Objectives: In the current study we ought to determine differential immune cell composition and heterogeneity in peripheral blood of SSc patients and its impact on disease severity and progression.

Methods: Mononuclear cells from blood of SSc patients with interstitial lung disease (ILD, $n=10)$ or No ILD $(n=10)$ and healthy controls $(n=10)$ were analysed by mass cytometry using a 36 marker (cell-surface and intracellular) panel to aid in identification of major PBMC lineages including T cells, B cells, monocytes and NK cells and their subsets. Transcriptome analysis (m-RNA sequencing) was performed on sorted T and B cell subsets. Unsupervised clustering of mass cytometry data was performed using in-house developed analysis software MARVIS This software combines dimension reduction and clustering steps to identify all possible cellular subsets. Further, custom $\mathrm{R}$ scripts helped in identifying nodes that were differentially expressed between the study groups and also phenotype of these nodes.

Results: Unsupervised clustering performed revealed significant differences in the frequencies of $T$ cell and $B$ cell subsets. Most strikingly we identify a 3 fold decrease in frequencies of Va7.2+CD161+mucosal associated invariant $\mathrm{T}$ cells (MAIT) in SSc patients and 2 fold increase in total B cells, particularly 
CD19 +CD27 naive cells. A subset of memory CD8 +T cell, expressing CXCR3 was found to be increased in SSc patients as compared to healthy controls. Transcriptome analysis of sorted B cell and T cell subsets showed decrease in genes related to survival and increased expression of apoptotic genes in CD4,CD8 T and MAIT cells from SSc patients. Genes related to exhaustion and leukocyte migration were highly expressed in T cells from patients.

Conclusions: This study provides an in depth analysis of systemic immune composition in SSc with the potential to delineate mechanisms of pathogenesis and identify diagnostic and/or therapeutic targets. This is the first demonstration of dysfunction of MAIT cells in SSc and further characterisation of their function in this context is required.

\section{REFERENCES:}

[1] Denton CP, Khanna D. 'Systemic sclerosis.' Lancet 2017;390 (10103):1685-1699

[2] Fuschiotti P. Current perspectives on the role of CD8+ T cells in systemic sclerosis. Immunol Lett 2017.

[3] Liu M, et al. New insights into CD4(+) T cell abnormalities in systemic sclerosis.' Cytokine Growth Factor Rev 2016;28:31-36.

[4] Sanges S, et al. 'Role of B cells in the pathogenesis of systemic sclerosis.' Rev Med Interne 2017;38(2):113-124.

Disclosure of Interest: None declared

DOI: 10.1136/annrheumdis-2018-eular.6082

\section{FRIDAY, 15 JUNE 2018}

\section{Navigating the world of digital health}

\section{OP0343-HPR AN ECONOMIC EVALUATION OF A TAILORED GUIDED INTERNET-BASED COGNITIVE BEHAVIOURAL INTERVENTION FOR PATIENTS WITH RHEUMATOID ARTHRITISAS AN ADDITION TO USUAL CARE}

M. Ferwerda ${ }^{1,2}$, S. van Beugen ${ }^{2}$, H. van Middendorp ${ }^{2}$, H. Visser ${ }^{3}$, H. Vonkeman ${ }^{4}$, M. Creemers ${ }^{5}$, P. van Riel ${ }^{6}$, W. Kievit ${ }^{7}$, A. Evers ${ }^{2} .{ }^{1}$ Medical Psychology, Radboud University Medical Centre, Nijmegen; ${ }^{2}$ Health, medical and neuropsychology, University of Leiden, Leiden; ${ }^{3}$ Rheumatology, Rijnstate hospital, Arnhem; ${ }^{4}$ Arhritis Centre Twente, Medisch Spectrum Twente and University of Twente, Enschede; ${ }^{5}$ Rheumatology, Jeroen Bosch Hospital, Den Bosch; ${ }^{6}$ Scientific institute for Quality of Healthcare; ${ }^{7}$ Radboud Institute for Health Sciences, Radboud University Medical Centre, Nijmegen, Netherlands

Background: Within the field of rheumatoid arthritis (RA), patients report decreased health-related quality of life (HRQoL) as a result of living with physical factors such as pain and psychological factors such as negative mood. As these factors are associated with the disease trajectory, health care utilisation, and workplace disability of patients, these factors lead to significant societal health expenses. In a recent randomised controlled trial, improvements in especially psychological functioning (e.g., depressed mood) were found by offering tailored, therapist guided cognitive behavioural therapy online. ${ }^{1}$ Although internet-based cognitive behavioural therapy holds promise for implementation and cost-reductions, scarce research is available on the cost-effectiveness of these treatments. Objectives: A cost-effectiveness study from a societal perspective was conducted alongside a randomised controlled trial on a tailored and therapist-guided internet-based cognitive behavioural intervention (ICBT) for patients with elevated levels of distress, as an addition to usual care alone in order to inform stake-holders on implementation of this treatment.

Methods: Data were collected at baseline/pre-intervention, 6 months/post-intervention, and three-monthly thereafter during one year follow-up. Effects were measured in quality-adjusted life years (QALYs) and costs from a societal perspective including healthcare sector costs (including healthcare use, medication, and intervention costs), patient travel costs for healthcare use, and costs associated with loss of labour.

Results: The intervention improved quality of life compared to usual care alone $(\triangle$ QALYs $=0.059)$, but also led to higher costs $(\Delta=€ 4.211,44)$, which reduced substantially when medication costs were left out of the equation ( $\Delta=€ 1.862,72$ ). Most $(93 \%)$ of the simulated ICERS were in the north-east quadrant, suggesting a high probability that the intervention is effective in improving HRQoL, but at a greater monetary cost for society compared to usual care alone.

Conclusions: A positive effect on quality-adjusted life years is seen in the intervention group compared to the control group. However, cost-ratios show that this comes at a greater cost to society. The substantial costs in this population are generated by medication costs, for which no group differences could be found. The cost-benefit ratio improves when the costs for medication are not taken into account.
Based on the effects for improvement of quality of life, implementation of the intervention is recommended, yet on the side of costs, further study is warranted.

\section{REFERENCE:}

[1] Ferwerda M, van Beugen S, van Middendorp H, Spillekom-van Koulil $S$ Donders ART, Visser H, Taal E, Creemers MCW, van Riel PCLM, Evers AWM. A tailored-guided internet-based cognitive-behavioural intervention for patients with rheumatoid arthritis as an adjunct to standard rheumatoogical care: Results of a randomized controlled trial. Pain 2017;158 (5):868-78. PMID: 28106666

Disclosure of Interest: None declared DOI: 10.1136/annrheumdis-2018-eular.5349

FRIDAY, 15 JUNE 2018

\section{The rheumatologist-orthopaedic surgeon connexion in secondary fracture prevention}

\section{OP0344 FRAME STUDY: THE FOUNDATION EFFECT OF REBUILDING BONE WITH ONE YEAR OF ROMOSOZUMAB LEADS TO CONTINUED LOWER FRACTURE RISK AFTER TRANSITION TO DENOSUMAB}

F. Cosman ${ }^{1}$, D.B. Crittenden ${ }^{2}$, S. Ferrari ${ }^{3}$, A. Khan ${ }^{4}$, N.E. Lane ${ }^{5}$, K. Lippuner ${ }^{6}$, T. Matsumoto ${ }^{7}$, C.E. Milmont ${ }^{2}$, C. Libanati ${ }^{8}$, A. Grauer ${ }^{2} .{ }^{1}$ Helen Hayes Hospital; Columbia University, West Haverstraw; New York; ${ }^{2}$ Amgen Inc., Thousand Oaks, CA, USA; ${ }^{3}$ Geneva University Hospital, Geneva, Switzerland; ${ }^{4}$ McMaster University, Hamilton, Ontario, Canada; ${ }^{5}$ UC Davis Medical Center, Sacramento, CA, USA; ${ }^{6}$ Osteoporosis Policlinic, Inselspital, Bern University Hospital and University of Bern, Bern, Switzerland; ${ }^{7}$ University of Tokushima, Tokushima, Japan; ${ }^{8}$ UCB Pharma, Brussels, Belgium

Objectives: Romosozumab (Romo), a sclerostin antibody, has a dual effect of increasing bone formation and decreasing bone resorption. In the FRAME study (NCT01575834), one year of Romo treatment resulted in large bone mineral density (BMD) increases at the lumbar spine (LS) and total hip (TH) versus placebo (Pbo). ${ }^{1}$ The differences between groups remained after all subjects transitioned to denosumab (DMAb) during the second year of study. Here, we further characterise the BMD gains seen during the FRAME study and examine the effect of building bone with Romo on fracture-risk reduction after transition to DMAb.

Methods: Subjects in FRAME were randomised to receive monthly Romo $210 \mathrm{mg}$ or Pbo for 12 months, after which all subjects received 6-monthly DMAb $60 \mathrm{mg}$ for an additional 12 months. Endpoints for the current analysis were mean change from baseline in BMD T-score, percentage of subjects with a BMD gain, and fracture incidence in the second year of the FRAME study, including new vertebral, clinical (nonvertebral and symptomatic vertebral), and other fracture categories.

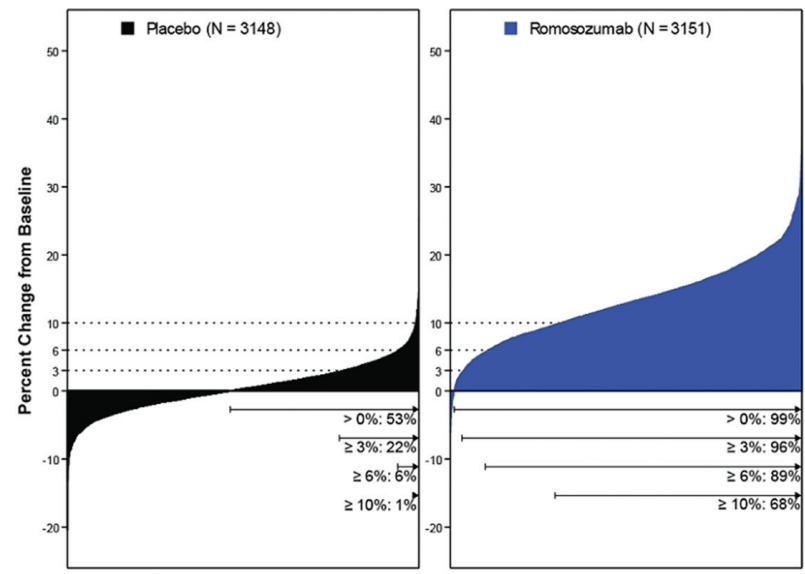

$\mathrm{N}=$ Number of subjects with values at baseline and at least one post-baseline visit at or before month 12 . Data are percent change in BMD from baseline to month 12 by individual subject at the lumbar spine. N's included subjects with baseline and $\geq 1$ postbaseline measurement; missing data were imputed by last observation carried
forward. $X$-axis represents each individual subject. Dotted horizontal lines reflect $3 \%, 6 \%$, and $10 \% \mathrm{BMD}$ gain from baseline to month 12. Lines with arrowheads below the $X$-axis represent the percent of subjects with the indicated BMD gains $(>0 \%, \geq 3 \%, \geq 6 \%$, and $\geq 10 \%$.

Abstract OP0344 - Figure 1 Percent change in lumber spine BMD from baseline to month 12 by individual subject 